\title{
A Secure Private Cloud Server Implementation for Data Storage
}

\author{
Amit Kumar Jha \\ Student (M.tech) \\ Department of CSE, \\ BUIT,Barkatullah University, \\ Bhopal, India
}

\author{
Divakar Singh \\ HOD \\ Department of CSE, BUIT, \\ Barkatullah University, \\ Bhopal, India
}

\begin{abstract}
This paper is the implementation of private cloud software as service server; centralized remote accessibility and data storage system without internet connection in Microsoft windows server $2 \mathrm{~K} 8$ with domain controlling .Migrating from a traditional model to the Cloud model reduce cost for enterprise customer. The main objectives of Saas provider are to minimize cost and to improve Customer Satisfaction Level (CSL)[13]
\end{abstract}

\section{INTRODUCTION}

Cloud computing has recently reached popularity in computer networking world which provide centralized data storage service , remote accessibility and many more cloud is very new, safe, and advanced technology it provides remote access service and centralized data storage service to the cloud consumers and its domain client in cloud. Cloud means a centralized domain hub which controls all the clients which are connected directly or in directly to the cloud server. It is based on virtualization which means we have no need to use any other special device to implement cloud server. Cloud is much secure and strong facility for corporate clients. computing provides a shared pool of resources, including data storage space, networks, computer processing power, and specialized corporate and user applications. In other words Cloud computing is a model for enabling convenient, on-demand network access to a shared pool of configurable computing resources (e.g., networks, servers, storage, applications, and services) that can be rapidly provisioned and released with minimal Management effort or service provider interaction. This cloud model promotes Availability and is composed of five essential characteristics, three service models, and four deployment models.

\section{LITRATURE REVIEW}

In this SaaS model, cloud providers install and operate application software in the cloud and cloud users access the software from cloud client. The cloud under do not manage the cloud infrastructure \& platform on which the application Is running. This eliminate the need to install and run the application on cloud user own computer simplifying maintenance and s upport. This is typically end user applica- tion delivered on demand over a network on a pay per use basis. The software required no client installation, just a browser and network connectivity Private cloud is a cloud in infrastructure build exclusively for a single organization, deployed within certain boundaries like firewall setting whether managed internally or by third-party and hosted internally . data a stored in the private cloud can only be shared amongst user of an organization. Private cloud has been a dopted by industries when security is something of primary concern such as finance and health care which have some of the most rigorous compliance requirements. Your business is your data and application. Therefore control and security are paramount.

\section{PROPOSED WORK}

In this paper we are implementing a cloud server with windows server $2 \mathrm{k} 8$ with the services OS Software as a service (SAAS) who can help their clients to use operating systems, with different different software's remotely and they can install in their personal computers, so they can store their data into cloud server safely and can use multiple software's without buying licensing services . And also they can store their private data in their private cloud without using internet services. In this paper we have implemented a private cloud software as a services server with data storage system for cloud clients in private network

\section{Previous algorithm to calculate time coast (TTL):}

Minimize $($ cost $)=$ VM Cost + Penalty Cost

Where Penalty Cost $=100$

Per Unit Time VMC cost large $=\mathrm{VM}$ Price large $\mathrm{X}$ Min $(\mathrm{VM}$ large $)=$ VM Price large

Client needs application software's and windows 32 bit application for their databases

$\mathrm{SQI}=\operatorname{respT}(\mathrm{SLA})-\operatorname{respT}($ actual $)=32 \mathrm{bit} / \mathrm{seconds}$

\section{EXPERIMENT METHODOLOGY}

To implement cloud server we have to install and configure Hyper-V with the following steps

1. Open Windows Firewall with Advance Security and click Inbound Rules.

2. Right-click Hyper-V Replica HTTP Listener (TCP-In) and click Enable Rule.

Enable the firewall rules for certificate-based authentication

1. Open Windows Firewall with Advance Security and click Inbound Rules.

2. Right-click Hyper-V Replica HTTPS Listener (TCP-In) and click Enable Rule.

For servers that are part of a failover cluster, run this Windows PowerShell cmdlet on any node in the cluster if you will be using Kerberos authentication for Replica. The cmdlet must be run by a user with administrative privileges.

get-clusternode | ForEach-Object \{Invoke-command computername \$_.name -scriptblock \{Enable-Netfirewallrule displayname "Hyper-V Replica HTTP Listener (TCP-In)" \} \}

For servers that are part of a failover cluster, run this Windows PowerShell cmdlet on any node in the cluster if you will be using certificate-based authentication for Replica. The cmdlet must be run by a user with administrative privileges. 
get-clusternode | ForEach-Object \{Invoke-command computername \$_.name -scriptblock \{Enable-Netfirewallrule displayname "Hyper-V Replica HTTPS Listener (TCP-In)" \} \}

Configure the Hyper-V Replica Broker

1.In Server Manager, open Failover Cluster Manager.

2.In the left pane, connect to the cluster, and while the cluster name is highlighted, click Configure Role in the Actions pane. The High Availability wizard opens

3. In the Select Role screen, select Hyper-V Replica Broker.

4. Complete the wizard, providing a NetBIOS name and IP address to be used as the connection point to the cluster (called a "client access point"). The Hyper-V Replica Broker is configured, resulting in a client access point name. Make a note of the client access point name for configuring Replica later on.

5. Verify that the Hyper-V Replica Broker role comes online successfully and can fail over between all nodes of the cluster. To do this, right-click the role, point to Move, and then click Select Node. Then, select a node, and then click OK..Windows Power Shell equivalent commands

The following Windows Power Shell cmdlet or cmdlets perform the same function as the preceding procedure. Enter each cmdlet on a single line, even though they may appear word-wrapped across several lines here because of formatting constraints. This example sequence of cmdlets will create a Hyper-V Replica Broker names "HVR-Broker" that uses the static IP address 192.168.1.5. All steps must be completed by a user with administrative privileges.

\section{$\$$ BrokerName $=$ "HVR-Broker"}

Add-ClusterServerRole -Name \$BrokerName -StaticAddress 192.168.1.5 Add-ClusterResource -Name "Virtual Machine Replication Broker" -Type "Virtual Machine Replication Broker"Group\$BrokerNameAdd-lusterResourceDependency

"Virtual Machine Replication Broker"\$Broker NameStartClusterGroup \$BrokerName create a self-signed certificate with Makecert.exe

Time coast (TTL) reduce algorithm: when we provide all applications and windows operating system to the client

Minimize $($ cost $)=\mathrm{VM}$ Cost + Penalty Cost;

Where Penalty Cost $=0$

Per Unit Time VMC cost large $=\mathrm{VM}$ Price large 0 Min $(\mathrm{VM} 0)=$ VM Price large X VMcost

Where VMcost $=0$

$\mathrm{SQI}=\operatorname{respT}(\mathrm{SLA})-\operatorname{respT}($ actual $)=32$ and $64 \mathrm{bit} / \mathrm{seconds}$

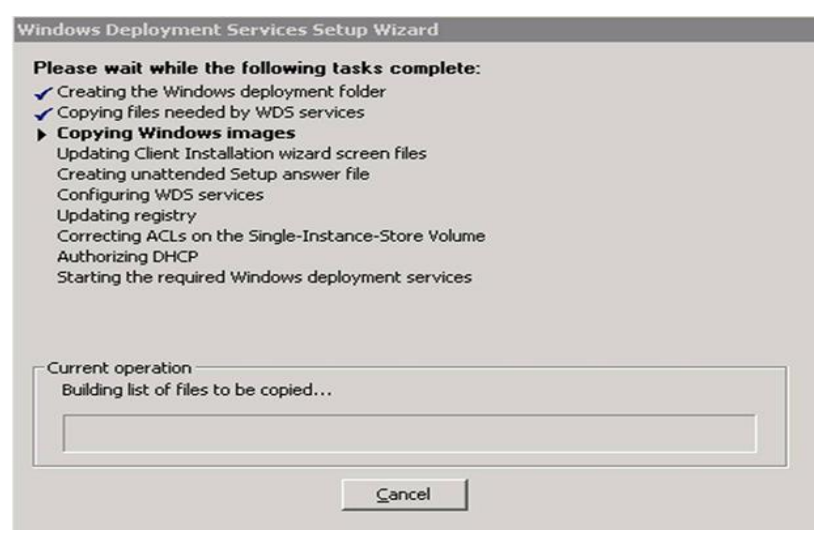

\section{RESULT ANALYSIS}

After this implementation process client can connect from cloud server remotely and can use its software as service facility, client can install operating systems, software from everywhere. Client can download software also, but cloud clients need to register on cloud server.All those software, windows applications and operating systems can be 32 bit or 64 bit. Which reduce the cost of VM and increases the time limits for all cloud clients?

\section{Previous}

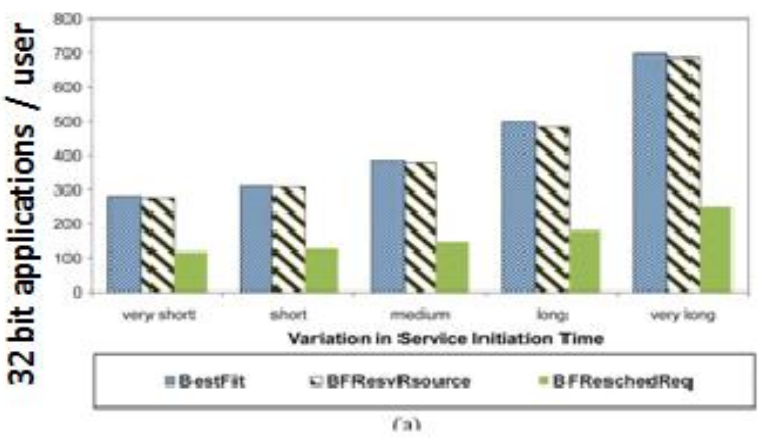

Fig .2 32 bit application usage Updated cost result: reducing cost and 64 bit software deployment for saving user time (TTL)

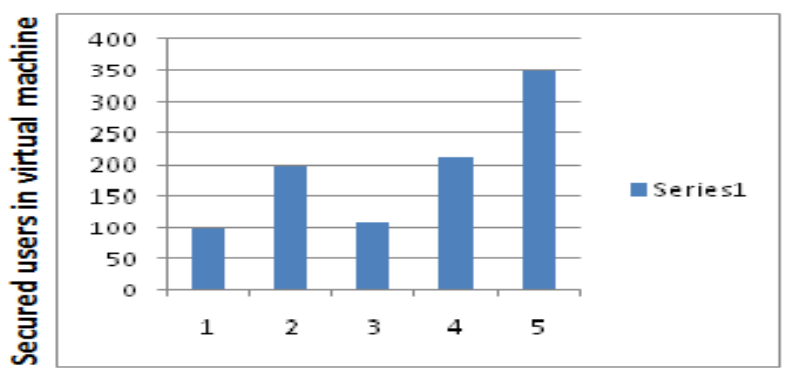

Fig 3. Secured users parameters Comparison chart after algorithum:

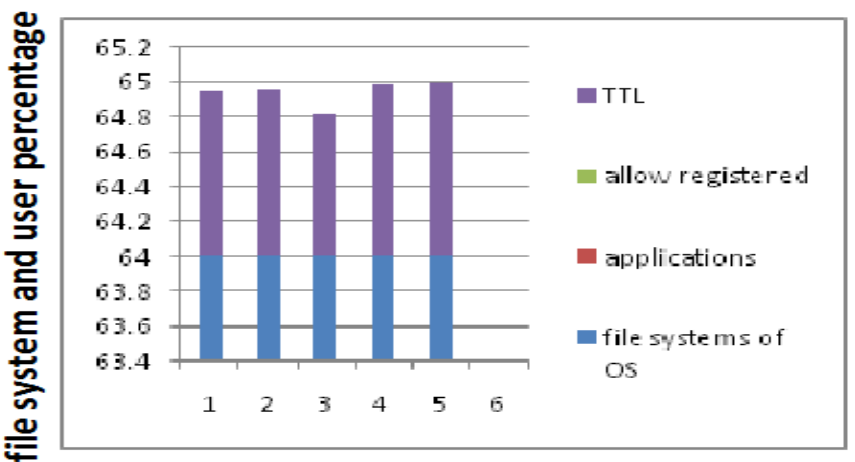

Fig.4 registerd users for 32 bit and 64 bit applications.

In previous result user can just use 32 bit applications from server. and after implementing algorithum user need to register first to get user id and password and user can also use 32 bit and 64 bit applications both and also can install 32 bit and 64 bit operating system remotely from server

Start $=>$ Run $=>$ Type the command MSTSC

Type $=>192.168 .1 .1$ (cloud servers ip address)

Username $=>$ Client 1

Password =>@\#passwd 


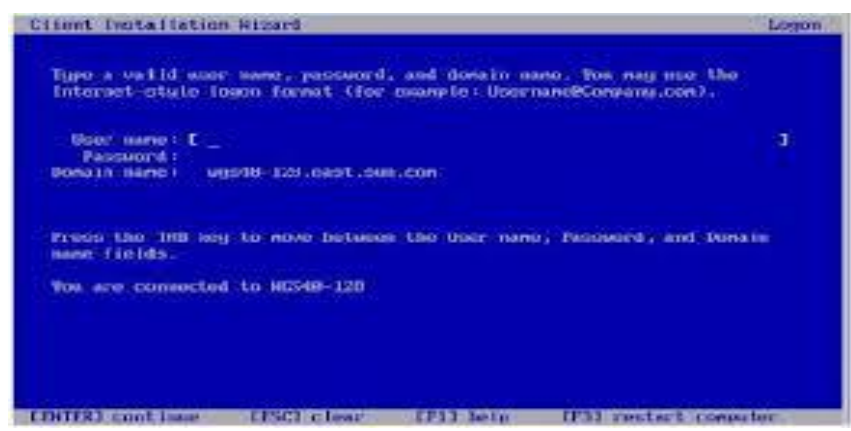

Fig 5. Client login window

\section{CONCLUSION:-}

After all these configuration client will directly connect to the cloud server and can access cloud client software as a service from server, server will provide operating system to the client and software's, with all these facilities client can also use or download software's from cloud server express to entire private cloud networks, server and to cloud clients and user can also use 32 bit and 64 bit applications both and also can install 32 bit and 64 bit operating system remotely from server

\section{FUTURE WORK}

After these privileges and process we can work further in future also for security purpose, we can use firewalls and advance security system, access control list and many for for further modifications and services deployment

\section{REFERENCES}

[1] A Secure Framework for User Data Storage in Public Cloud Systems using Symmetric Cryptography Reetika Singh1, Rajesh Tiwari2 1Research Scholar, Faculty of Engineering \& Technology, 2Faculty of Engineering \& Technology Department of Computer Science \& Engineering, Shri Shankaracharya College of Engineering \& Technology, Chhattisgarh Swami Vivekananda Technical University, Bhilai - 490006, Chhattisgarh, INDIA

[2] Cloud computing: state-of-the-art and research challenges Qi Zhang. Lu Cheng. Raouf Boutaba

[3] Cloud Computing - Issues, Research and Implementations, Mladen A. Vouk Department of Computer Science, North Carolina State University, Raleigh, North Carolina, USA

[4] Cloud Computing and Emerging IT Platforms: Vision, Hype, and Reality for Delivering Computing as the 5th Utility ,Rajkumar Buyya 1,2, Chee Shin Yeo 1, Srikumar Venugopal 1, James Broberg 1, and Ivona Brandic 3,1 Grid d Computing and Distributed Systems (GRIDS) Laboratory Department of Computer Science and Software Engineering The University of Melbourne, Australia Email: \{raj, csyeo, srikumar, brobergj\}@csse.unimelb.edu.au ,2 Manjrasoft Pty Ltd, Melbourne, Australia.

[5] Cloud Computing Nariman Mirzaei (nmirzaei@indiana.edu) Fall 2008.,
[6] FOSTER, I., ZHAO, Y., RAICU, I. and LU, S. 2008. Cloud Computing and Grid Computing 360-Degree Compared. In Grid Computing Environments Workshop (GCE '08), Austin, Texas, USA, November 2008, 1-10

[7] Corbató, F. J., Saltzer, J. H., and Clingen, C. T. 1972. Multics: the first seven years. In Proceedings of the May 1618, 1972, Spring Joint Computer Conference, Atlantic City, New Jersey, May 1972, 571-583.

[8] BUYYA, R., YEO, C. and VENUGOPAL, S. 2008. MarketOriented Cloud Computing: Vision, Hype, and Reality for Delivering IT Services as Computing Utilities. In High Performance Computing and Communications, 2008. HPCC '08. 10th IEEE International Conference on, 5-13

[9] Implementing and operating an internet scale distributed application using service oriented architecture principles and cloud computing infrastructure. In iiWAS '08: Proceedings of the 10th International Conference on Information Integration and Web-based Applications \& Services, 417-421. CHANG, M., HE, J., and E. Leon, "Service-Orientation in the Computing Infrastructure," 2006, pp. 27-33. [26] SEDAYAO, J. 2008

[10] PROVIDING A SECURE DATA FORWARDING IN CLOUD STORAGE SYSTEM USING THRESHOLD PROXY RE-ENCRYPTION SCHEME. S.Poonkodi1, V.Kavitha2, K.Suresh3 ,1,2Assistant Professor, Information Technology, Karpaga Vinayaga College of Engineering \& Technology, Kanchipuram Dt, Tamil Nadu, India 3Assistant Professor, Computer Science \& Engineering, KCG College of Technology, Chennai, Tamil Nadu, India Email Address: rkpoonkodi@gmail.com,avija2000@yahoo.com,sureshvk.200 8@gmail.com.International Journal of Emerging Technology and Advanced Engineering Website: www.ijetae.com (ISSN 2250-2459 (Online), An ISO 9001:2008 Certified Journal, Volume 3, Special Issue 1, January 2013) International Conference on Information Systems and Computing (ICISC2013), INDIA.

[11] Secure Framework for Data Storage from Single to Multi clouds in Cloud NetworkingB.Sujana1, P.Tejaswini2, G.Srinivasulu3, Sk.Karimulla41,2,3,4 QUBA COLLEGE OF ENGINEERING \& TECH, NELLORE. International Journal of Emerging Trends \& Technology in Computer Science (IJETTCS),Web Site: www.ijettcs.org Email: editor@ijettcs.org, editorijettcs@gmail.com,Volume 2, Issue 2, March - April 2013 ISSN 2278-6856.

[12] Implementation of Data Security in Cloud Computing G. Jai Arul Jose1, C. Sajeev2 1,2 Research Scholar, Sathyabama University, Chennai, INDIA. International Journal of P2P Network Trends and Technology- July to Aug Issue 2011ISSN: 2249-2615.

[13] SLA-based resource provisioning for hosted software-as-aservice application in cloud computing environments.by linlin Wn,saurabh kumar garg,steve verteeg and rajkumar Buyya.IEEE vol-7,no 3,july-september 2014 